S. Kwok, M. Dopita, and R. Sutherland, eds.

\title{
NIR Spectroscopy of IRAS 16115-5044
}

\author{
David T.F Weldrake, Peter R. Wood \\ Research School of Astronomy \& Astrophysics, Australian National \\ University \\ Griet C. Van de Steene \\ Royal Observatory of Belgium
}

IRAS 16115-5044 has been identified as a possible post-AGB star on the basis of its position in the IRAS 2-colour diagram (van der Veen et al. 1989). A study by Van de Steene and Pottasch (1993) failed to detect any radio-continuum flux from the object, suggesting it is still in the pre-planetary nebula phase, that is, the central star is not yet hot enough to ionize the circumstellar shell.

The central star is bright in the infrared $(K=4.9)$ but faint in the visible which we attribute to an optically thick circumstellar shell $(\mathrm{J}-\mathrm{K}=2.61$, van der Veen et al. 1989). Oudmaijer et al. (1995) obtained a K-band spectrum of IRAS 16115-5044 longward of 2.2 microns, and did not detect any discernable features.

We have obtained near-infrared I, J, H and K-band spectra of IRAS 161155044 at a resolution $\lambda / \Delta \lambda$ of 1100 , using CASPIR attached to the ANU $2.3 \mathrm{~m}$ telescope at Siding Spring Observatory. The K-band spectrum is shown in Figure 1. IRAS 16115-5044 clearly displays emission lines from the hydrogen Paschen and Brackett series as well as from the metal ions FeII, MgII and NaI. This we attribute to the presence of a stellar wind. The P-Cygni profiles of the emission lines indicate an outflow velocity of $\mathrm{V}_{\exp } \sim 300 \mathrm{~km} \mathrm{~s}^{-1}$. From the Hband Paschen photospheric absorptionlines, we deduce a spectral type B4I. The width of these photospheric lines is typical of luminosity class I, a result which seems to confirm the post-AGB nature of this object.

$\mathrm{P} \beta$ imaging displays no extension down to an effective size of $0.3^{\prime \prime}$, allowing upper limits on the effective size of the outflow region to be made. Assuming an intrinsic luminosity of $5000 L_{\odot}$, typical of post-AGB stars at this stage in their evolution, a distance of $2 \mathrm{kpc}$ is derived. The line emission region is therefore very compact $\left(<9 \times 10^{15} \mathrm{~cm}\right)$ : the stellar wind would pass through this region in $<10 \mathrm{yr}$.

The initial conclusion is that this object is an extremely young and compact post-AGB star just beginning the process of planetary nebula formation. Further observations will be carried out at higher resolution, to fully understand the nature of this object. 


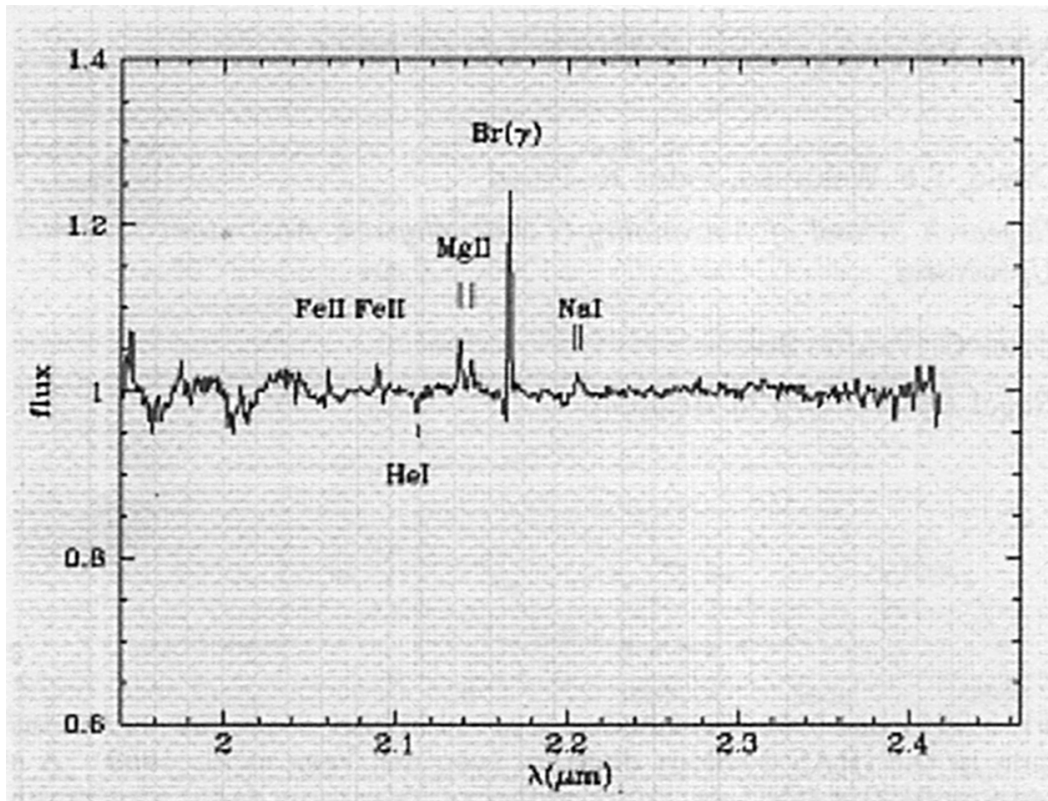

Figure 1. The K-band spectrum for IRAS 16115-5044, displaying emission lines of $\mathrm{Br} \gamma, \mathrm{FeII}, \mathrm{MgII}$ and $\mathrm{NaI}$.

\section{References}

Oudmaijer R.D, Waters L.B.F.M, van der Veen W.E.C.J, Geballe T.R, 1995, A\&A 299, 69

van der Veen W.E.C.J, Habing H.J, Geballe T.R, 1989, A\&A 226, 108

Van de Steene G.C, Pottasch S.R., 1993, A\&A 274, 895 